\title{
Number of Patients per Cohort and Sample Size Considerations Using Dose Escalation with Overdose Control
}

\author{
Mourad Tighiouart and André Rogatko \\ Samuel Oschin Comprehensive Cancer Institute, 8700 Beverly Boulevard, Los Angeles, CA 90048, USA \\ Correspondence should be addressed to Mourad Tighiouart, mourad.tighiouart@cshs.org
}

Received 29 June 2012; Accepted 15 September 2012

Academic Editor: Yichuan Zhao

Copyright (C) 2012 M. Tighiouart and A. Rogatko. This is an open access article distributed under the Creative Commons Attribution License, which permits unrestricted use, distribution, and reproduction in any medium, provided the original work is properly cited.

\begin{abstract}
The main objective of cancer phase I clinical trials is to determine a maximum tolerated dose (MTD) of a new experimental treatment. In practice, most of these trials are designed so that three patients per cohort are treated at the same dose level. In this paper, we compare the safety and efficiency of trials using the escalation with overdose control (EWOC) scheme designed with three or only one patient per cohort. We show through simulations that the number of patients per cohort does not impact the proportion of patients given therapeutic doses, safety of the trial, and efficiency of the estimate of the MTD. Additionally, we present guidelines and tabulated values on the number of patients needed to design a phase I cancer clinical trial using EWOC to achieve a given accuracy of the estimate of the MTD.
\end{abstract}

\section{Introduction}

Cancer phase I clinical trials are small studies whose main objective is to determine a maximum tolerated dose (MTD) of a new experimental drug or combination of known drugs for use in a phase II trial. Patients are typically accrued to the trial sequentially in cohorts of size $m$ and dose level assignment to a given cohort of patients is dependent upon the dose levels and toxicity outcomes of the previously treated cohorts of patients. A large number of statistical methodologies which account for the sequential nature of the data generated by such designs have been proposed in the literature, see $[1,2]$ for a comprehensive review of such methods. In particular, the continual reassessment method (CRM) proposed by [3] and its modifications [4-8] and escalation with overdose control (EWOC) described in [915] are Bayesian adaptive designs that produce consistent sequences of doses and can be easily implemented in practice using published tutorials and free interactive software, see, for example, [16-19]. 
The work we present here has been motivated by the frequent requests by clinicians and review committees at Cancer Center Institutions the authors worked at on (1) the number of patients that should be included in each cohort, and (2) the number of patients required to conduct a phase I cancer clinical trial. Denote by ${ }^{m} P$ a design that treats patients in successive cohorts of size $m$ simultaneously at the same dose level. For a given fixed number of patients in the trial, an advantage of an ${ }^{m} P$ design with $m>1$ over a ${ }^{1} P$ design is a shorter time of completion of the trial. However, it is not clear how the two designs compare with respect to safety of the trial and efficiency of the estimate of the MTD using EWOC. Goodman et al. [5] argue for the use of more than one patient per dose level in a modified version of the CRM to reduce the duration of the trial and toxicity incidence associated with the original CRM. In this paper, we compare a ${ }^{3} P$ design with a ${ }^{1} P$ design in terms of the number of patients given therapeutic doses, that is, doses in a neighborhood of the "true" MTD. In addition, safety of the trial and efficiency of the estimate of the MTD will be compared using extensive simulations.

The number of patients that are enrolled in a cancer phase I clinical trial is typically between 12 and 40 and trial duration depends on the study design and length of the study cycle to resolve toxicity outcome. An increasing number of clinicians we work with inquire about the number of patients they need to accrue in order to estimate the MTD with an acceptable degree of accuracy. We are not aware of any published methodologies for sample size determination (SSD) in cancer phase I clinical trials based on power calculation or precision of some Bayes estimates using either frequentist or Bayesian adaptive designs. As a point of fact, most sample size recommendations are based on prespecified stopping rules, see, for example, [20] on selecting the number of patients by considering different stopping rules using the CRM. Lin and Shih [21] and Ivanova [22] describe sample size recommendations based on the expected number of patients allocated to each dose selected from a set of prespecified dose levels.

In this paper, we address the SSD problem using the traditional approach; we estimate the sample size based on a desired accuracy of the Bayes estimate on the average. Specifically, we seek the smallest number of patients so that the posterior variance of the MTD on the average over all possible trials is no more than a specified margin. This procedure is not based on a specific stopping rule and consequently preserves the coherent nature of EWOC, see [15] for the coherence EWOC.

This paper is organized as follows. Section 2 describes dose escalation with overdose control using cohorts of size $m$. In Section 3, we present two criteria to sample size determination in this Bayesian setting. Comparisons of designs that treat cohorts of size $m>1$ simultaneously over the ones that treat one patient at a time are presented in Section 4. In that section, we also give tabulated values relating the number of patients on the trial and the corresponding average posterior variance and length of the highest posterior density interval. Section 5 contains some concluding remarks and discussion.

\section{2. ${ }^{m} P$ Design Using EWOC}

EWOC is a Bayesian adaptive design permitting precise determination of the MTD while directly controlling the likelihood of an overdose. It is the first statistical method to directly incorporate formal safety constraints into the design of cancer phase I clinical trials. Zacks et al. [10] and Tighiouart and Rogatko [15] discuss statistical properties and coherence of the method, and a comparison of EWOC with alternative phase I design methods is given 
in [9]. Babb and Rogatko [11] provide a summary of Bayesian phase I design methods and Tighiouart et al. [12] studied the performance of EWOC under a richer class of prior distributions for the model parameters. The defining property of EWOC is that the expected proportion of patients treated at doses above the MTD is equal to a specified value $\alpha$, the feasibility bound. This value is selected by the clinician and reflects his/her level of concern about overdosing. Zacks et al. [10] showed that among designs with this defining property, EWOC minimizes the average amount by which patients are underdosed. This means that EWOC approaches the MTD as rapidly as possible, while keeping the expected proportion of patients overdosed less than the value $\alpha$. Zacks et al. [10] also showed that, as a trial progresses, the dose sequence defined by EWOC approaches the MTD (i.e., the sequence of recommended doses converges in probability to the MTD). Eventually, all patients beyond a certain time would be treated at doses sufficiently close to the MTD.

EWOC has been used to design over a dozen of phase I studies approved by the Research Review Committee and the Institute Review Board of the Fox Chase Cancer Center, Philadelphia, Winship Cancer Institute, Atlanta, and Cedars Sinai Medical Center, Los Angeles (see [23-29] for some of the published trials). following:

We adopt the-logistic-based model to represent the dose-toxicity relationship the

$$
\operatorname{Prob}(\mathrm{DLT} \mid \text { Dose }=x)=\frac{\exp \left(\beta_{0}+\beta_{1} x\right)}{1+\exp \left(\beta_{0}+\beta_{1} x\right)}
$$

where $\left(\beta_{0}, \beta_{1}\right) \in(-\infty, \infty) \times(0, \infty)$ so that the probability of dose limiting toxicity (DLT) is an increasing function of dose. The MTD $\gamma$ is defined as the dose expected to produce DLT in a specified proportion $\theta$ of patients. Let $\rho_{0}$ be the probability of a DLT at the starting dose. To facilitate interpretation of model parameters by the clinicians, we further parameterize model (2.1) in terms of $\left(\rho_{0}, \gamma\right)$, see $[9,12]$ for more details. Suppose we plan to enroll $n$ patients in the trial in cohorts of size $m$. Dose levels in the trial are selected in the interval $\left[X_{\min }, X_{\max }\right]$ and an ${ }^{m} P$ design proceeds as follows. We first specify prior distributions for $\rho_{0}$ and $\gamma$. Then, the first cohort of $m$ patients receives the dose $x_{1}=X_{\min }$. Let $d_{1}$ be the number of toxicities observed among the first $m$ patients. The likelihood given the observed data thus far is

$$
L_{1}\left(\rho_{0}, \gamma \mid D_{1}\right)=p\left(\rho_{0}, \gamma, x_{1}\right)^{d_{1}}\left(1-p\left(\rho_{0}, \gamma, x_{1}\right)\right)^{m-d_{1}},
$$

where

$$
p\left(\rho_{0}, \gamma, x_{1}\right)=\frac{\exp \left\{\ln \left[\rho_{0} /\left(1-\rho_{0}\right)\right]+\ln \left[\theta\left(1-\rho_{0}\right) / \rho_{0}(1-\theta)\right]\left(x_{1} / \gamma\right)\right\}}{1+\exp \left\{\ln \left[\rho_{0} /\left(1-\rho_{0}\right)\right]+\ln \left[\theta\left(1-\rho_{0}\right) / \rho_{0}(1-\theta)\right]\left(x_{1} / \gamma\right)\right\}}
$$

and $D_{1}=\left\{\left(x_{1}, d_{1}\right)\right\}$. Let $\Pi_{1}(x)$ be the marginal posterior cumulative distribution function (cdf) of the MTD $\gamma$ given $D_{1}$. The second cohort of $m$ patients receives the dose $x_{2}=\Pi_{1}^{-1}(\alpha)$ so that the posterior probability of exceeding the MTD is equal to the feasibility bound $\alpha$. In 
general, the likelihood of the data after observing the toxicity outcomes of the $i$ th cohort of $m$ patients is

$$
L_{i}\left(\rho_{0}, \gamma \mid D_{i}\right)=\prod_{j=1}^{i} p\left(\rho_{0}, \gamma, x_{j}\right)^{d_{j}}\left(1-p\left(\rho_{0}, \gamma, x_{j}\right)\right)^{m-d_{j}},
$$

where $x_{j}$ is the dose assigned to the $j$ th cohort of $m$ patients, $p\left(\rho_{0}, \gamma, x_{j}\right)$ is given by (2.3) with $x_{1}$ replaced by $x_{j}$, and $D_{i}=\left\{\left(x_{1}, d_{1}\right), \ldots,\left(x_{i}, d_{i}\right)\right\}$. The $(i+1)$ st cohort of $m$ patients receives the dose $x_{i+1}=\Pi_{i}^{-1}(\alpha)$ where $\Pi_{i}(x)$ is the marginal posterior cdf of $\gamma$ given $D_{i}$. This process is repeated until a total of $k$ cohorts are enrolled in the trial. This completes the description of an ${ }^{m} P$ design. For a given sample size $n$, we propose to compare the performance of a ${ }^{1} P$ with a ${ }^{3} P$ design by estimating the percent of patients treated within a neighborhood of the true MTD. Other comparisons include safety and efficiency of the estimate of the MTD under the two designs.

\section{Sample Size Determination}

An increasing number of clinicians inquire about the number of patients they need to accrue in the design of cancer phase I trials to achieve a specific goal. Sample size recommendation based on the expected number of patients treated at each dose level in " $3+3$ " designs and $A+B$ designs have been studied in [21, 22], respectively. However, these methods apply to a prespecified set of discrete doses and it is not clear how they can be applied to continuous doses. Unlike the frequentist approach, there is no consensus on a specific Bayesian method for the SSD problem, see Adcock [30] for a review of Bayesian approaches. In this paper, we present numerical results based on the posterior variance of the MTD and highest posterior density (HPD) interval, see [31].

Denote by $\operatorname{Var}\left(\gamma \mid D_{n}\right)$ the posterior variance of the MTD given that $n$ patients have been accrued to the trial. The first criterion is to find the smallest $n$ that satisfies

$$
E_{D_{n}}\left[\operatorname{Var}\left(r \mid D_{n}\right)\right] \leq \eta
$$

where the above expectation is taken with respect to the marginal distribution of the data and $\eta$ is specified by the clinician. In other words, we require an estimate of the MTD within a given accuracy as measured by the posterior variance on the average overall possible trials. In the second criteria, we seek the smallest $n$ such that

$$
E_{D_{n}}\left[l\left(D_{n}\right)\right] \leq d,
$$

where $l\left(D_{n}\right)$ is the length of the HPD interval $\left(a, a+l\left(D_{n}\right)\right)$ determined by the constraint on the coverage probability

$$
P\left[\gamma \in\left(a, a+l\left(D_{n}\right)\right) \mid D_{n}\right]=1-\alpha_{1} .
$$

This is also known as the average length criteria (ALC) because for each realization of a trial $D_{n}$, the corresponding HPD interval is determined by (3.3) and the lengths of these 
HPD intervals are averaged out with respect to the marginal distribution of the data in (3.2). The tolerance values of the average length of the HPD interval $d$ and coverage probability $1-\alpha_{1}$ are prespecified by the clinician. Since both the posterior distribution of the MTD and marginal distribution of the data are intractable, Monte Carlo averages were used to estimate the left hand sides of (3.1) and (3.2). Details on the computation of $\operatorname{Var}\left(\gamma \mid D_{n}\right)$ and $l\left(D_{n}\right)$ can be found in $[9,18]$.

\section{Numerical Results}

The simulation results presented below all assume that the feasibility bound $\alpha=0.25$ and that the dose levels are standardized so that the starting dose for each trial is $x_{1}=0$ and all subsequent dose levels are selected from the unit interval. Independent uniform prior distributions were put on the parameters $\rho_{0}$ and $\gamma$ on the intervals $[0, \theta],[0,1]$, respectively.

\subsection{Comparison of Designs ${ }^{3} P$ with ${ }^{1} P$}

We simulate trials under different scenarios corresponding to different values of $\rho_{0}$ and $\gamma$. For the ${ }^{1} P$ design, the first patient receives dose 0 and the next dose $x_{2}$ is determined as described in Section 2. The second response $y_{2}$ is then generated from the logistic model (2.3). This process is repeated until a trial of $n$ patients is generated. The same process applies to the ${ }^{3} P$ design except that 3 patients will be given the same dose at each stage of the trial and 3 responses are generated from model (2.3) independently instead of 1 . Since $0 \leq \rho_{0} \leq \theta$ and $0 \leq r \leq 1$, we considered 12 scenarios corresponding to combinations of three values of $\rho_{0},\{\theta / 4, \theta / 2,3 \theta / 4\}$ with four values of $\gamma, 0.2,0.4,0.6$, and 0.8 . We will refer to $\theta / 4, \theta / 2$, $3 \theta / 4$ as low, intermediate, and high values for $\rho_{0}$, respectively. Similarly, 0.2 and 0.4 will be referred to as low values for the MTD $\gamma$ and 0.6 and 0.8 as high values. The same value $\theta=0.3$ was used in all simulations. For each design, each sample size $n=12,18,24,30$, and each combination of $\left(\rho_{0}, \gamma\right)$, we simulated 5000 trials and calculated the proportions of patients given therapeutic doses, that is, doses in an $\varepsilon$-neighborhood of the true MTD, for $\varepsilon=0.05,0.1,0.15,0.2$.

Table 1 gives the estimated proportions of patients given doses in an $\varepsilon$-neighborhood of the true MTD under designs ${ }^{1} P$ and ${ }^{3} P$ and the difference in these proportions between the two designs for low values of the true MTD $\gamma$ and different sample sizes. Table 2 gives the corresponding estimates for high values of the true MTD and Table 3 gives the average of these estimates across the 12 combination of $\left(\rho_{0}, \gamma\right)$. For low values of the true MTD, design ${ }^{1} P$ assigns more patients to doses near the MTD than design ${ }^{3} P$ in general and the difference can be as high as $16 \%$ for $\varepsilon=0.05,\left(\rho_{0}, \gamma\right)=(0.4,0.075)$, and $n=12$. For high values of the MTD, Table 2 shows that design ${ }^{1} P$ always assigns more patients to doses near the MTD than design ${ }^{3} P$ and the highest difference is about $16 \%$ for $\varepsilon=0.2,\left(\rho_{0}, \gamma\right)=(0.6,0.075)$, and $n=12$. The estimated difference in the proportions of patients given doses in an $\varepsilon$ neighborhood of the true MTD between the ${ }^{1} P$ design and ${ }^{3} P$ design averaged across the 12 entertained scenarios for $\left(\rho_{0}, \gamma\right)$ for different sample sizes show that the proportion of patients given therapeutic doses under design ${ }^{1} P$ is always greater than the corresponding proportion under design ${ }^{3} \mathrm{P}$, the largest of these differences is about $5 \%$. The practical impact of this difference is unimportant because of the relatively small number of patients involved in phase I cancer clinical trials. In Tables 4 and 5, we present differences in (i) the proportions of patients exhibiting DLT, (ii) the proportions of patients given doses above the "true" MTD, 


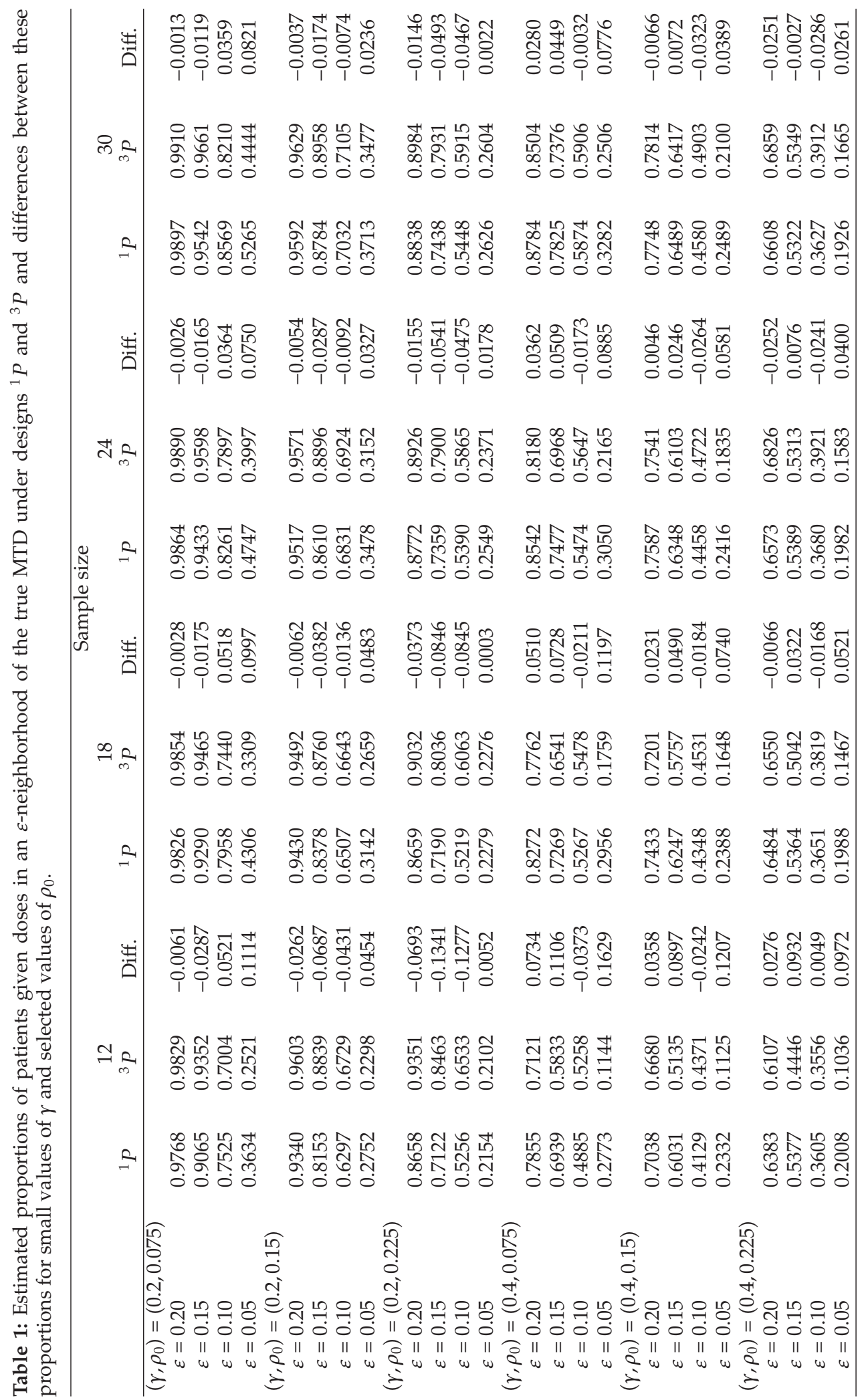


Journal of Probability and Statistics

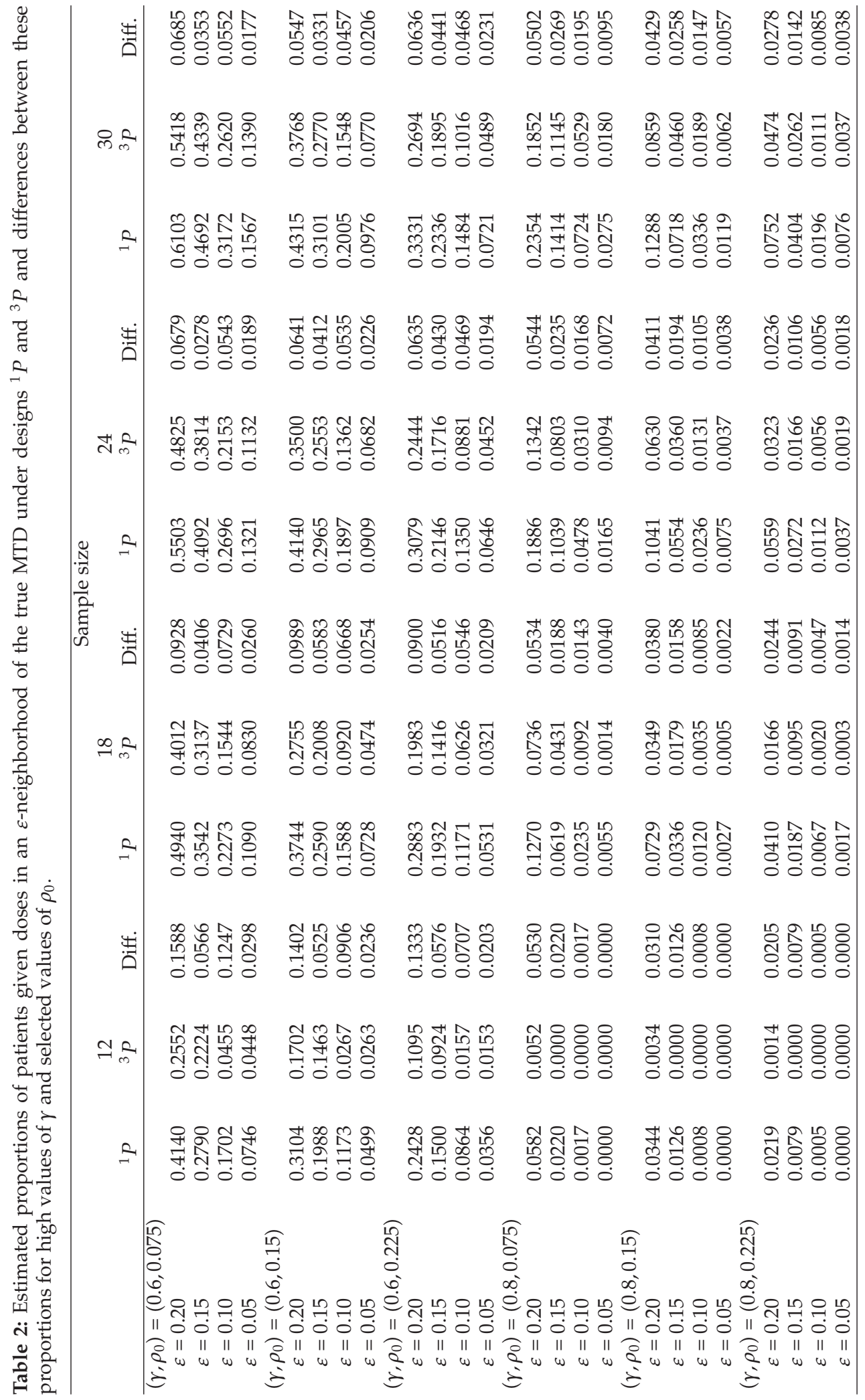


Table 3: Estimated proportions of patients given doses in an $\varepsilon$-neighborhood of the true MTD under designs ${ }^{1} \mathrm{P}$ and ${ }^{3} \mathrm{P}$ and differences between these proportions on the average.

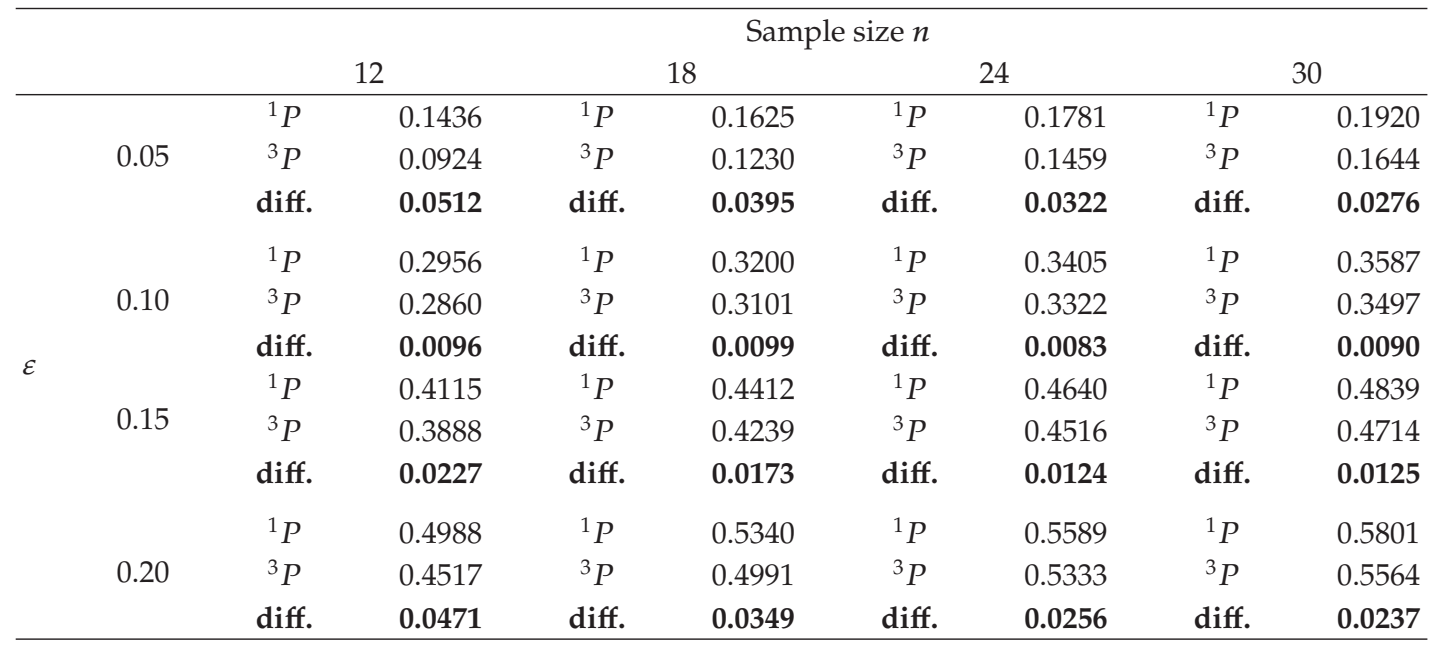

(iii) the bias, and (iv) the mean square error between the ${ }^{1} P$ and ${ }^{3} P$ designs. Table 6 gives the average values of these statistics, averaged across the 12 entertained scenarios for $\left(\rho_{0}, \gamma\right)$. Based on (i) and (ii), the results indicate that the two designs are equally safe and that no practical gain is achieved in terms of the efficiency of the estimate of the MTD according to (iii) and (iv). From an ethical point of view, we recommend the ${ }^{1} P$ design to prevent the occurrence of three simultaneous DLTs if we were to use the ${ }^{3} P$ design. This should be discussed with the clinician after assessing the importance of the length of the trial.

\subsection{Sample Size Determination}

In this section, we present tabulated values for average posterior standard deviation of the MTD and average length HPD interval that are achieved for even sample sizes $n=6, \ldots, 40$ and selected values of $\theta$, the target probability of DLT. Table 7 summarizes the results for $\theta=0.3$. For a given sample size $n$, each entry in the table was calculated according to the following algorithm:

Set $j=1$.

(i) Generate $\left(\rho_{0, j}, \gamma_{j}\right) \sim$ Uniform $[0, \theta] \times[0,1]$ and independently.

(ii) Simulate a trial of $n$ patients $D_{n, j}$ according to the EWOC algorithm described in Section 4.1 with $\left(\rho_{0, j}, r_{j}\right)$ as the true model parameters.

(iii) Calculate the posterior variance $\operatorname{Var}\left(\gamma \mid D_{n, j}\right)$ and $\operatorname{HPD}\left(a_{j}, a_{j}+l\left(D_{n, j}\right)\right)$ using (3.3).

(iv) Repeat steps (i)-(iii) for $j=2, \ldots, M$. 
Journal of Probability and Statistics

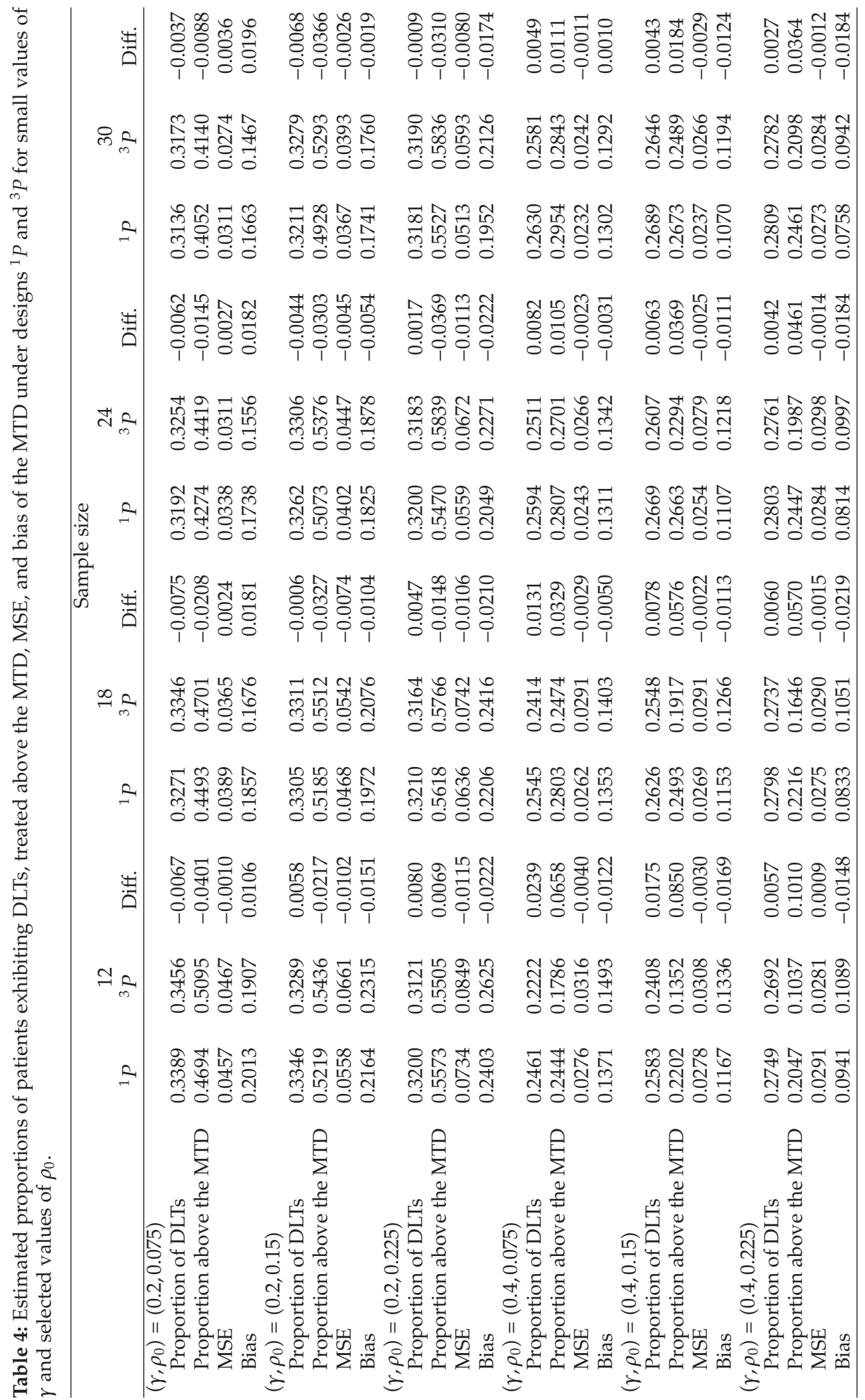




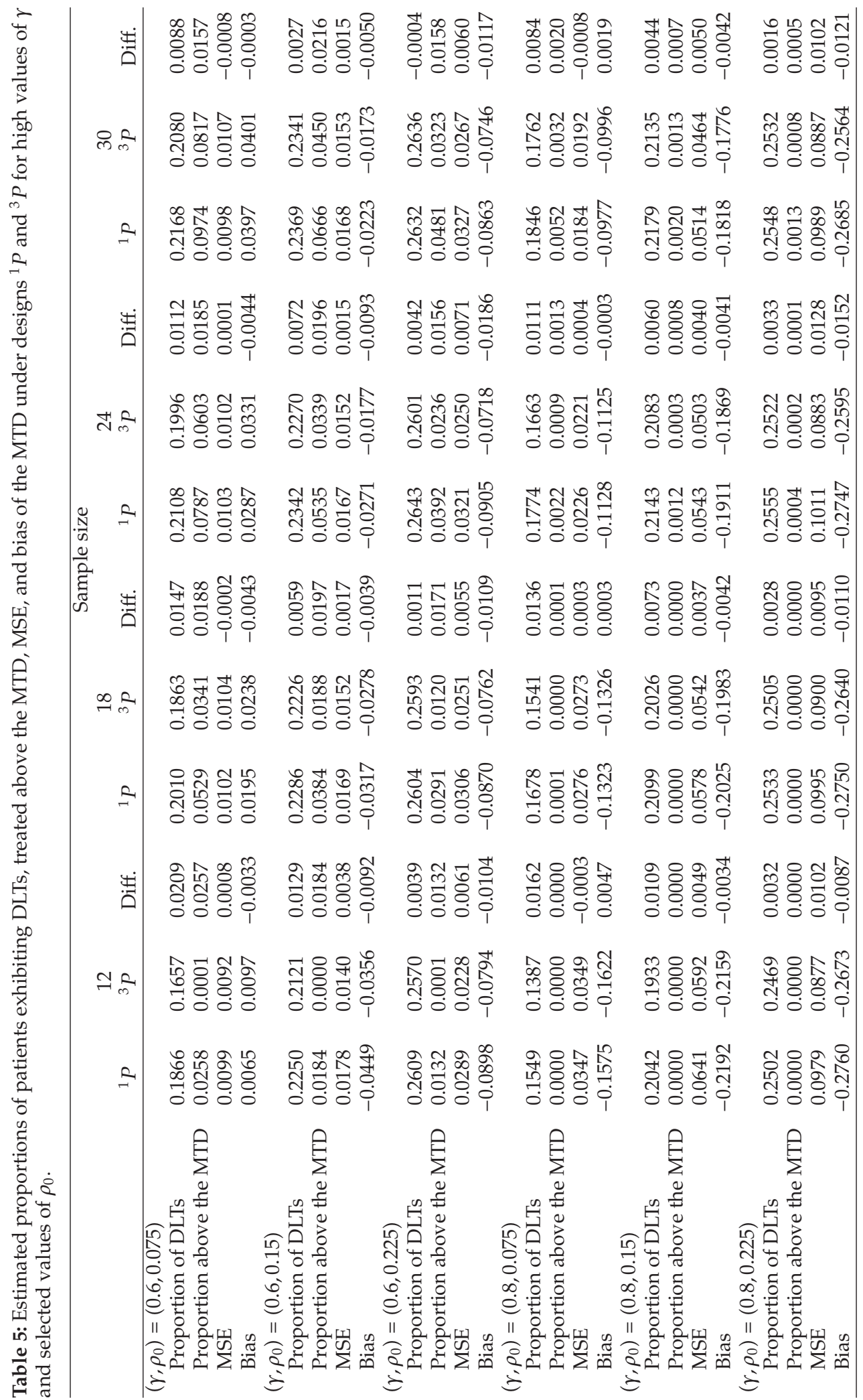


Table 6: Estimated proportions of patients exhibiting DLTs, treated above the MTD, MSE, and bias of the MTD under designs ${ }^{1} P$ and ${ }^{3} P$ and differences between these proportions on the average.

\begin{tabular}{|c|c|c|c|c|c|c|c|c|}
\hline & \multicolumn{8}{|c|}{ Sample size $n$} \\
\hline & \multicolumn{2}{|c|}{12} & \multicolumn{2}{|c|}{18} & \multicolumn{2}{|c|}{24} & \multicolumn{2}{|c|}{30} \\
\hline & ${ }^{1} P$ & 0.2546 & ${ }^{1} P$ & 0.2580 & ${ }^{1} P$ & 0.2607 & ${ }^{1} P$ & 0.2616 \\
\hline \multirow[t]{2}{*}{ Proportion of DLTs } & ${ }^{3} P$ & 0.2444 & ${ }^{3} P$ & 0.2523 & ${ }^{3} P$ & 0.2563 & ${ }^{3} P$ & 0.2595 \\
\hline & diff. & 0.0102 & diff. & 0.0057 & diff. & 0.0044 & diff. & 0.0021 \\
\hline \multirow{3}{*}{ Proportion above the MTD } & ${ }^{1} P$ & 0.1895 & ${ }^{1} P$ & 0.2001 & ${ }^{1} P$ & 0.2040 & ${ }^{1} P$ & 0.2067 \\
\hline & ${ }^{3} P$ & 0.1685 & ${ }^{3} P$ & 0.1888 & ${ }^{3} P$ & 0.1984 & ${ }^{3} P$ & 0.2029 \\
\hline & diff. & 0.0210 & diff. & 0.0113 & diff. & 0.0056 & diff. & 0.0038 \\
\hline \multirow{3}{*}{ MSE } & ${ }^{1} P$ & 0.0427 & ${ }^{1} P$ & 0.0394 & ${ }^{1} P$ & 0.0371 & ${ }^{1} P$ & 0.0351 \\
\hline & ${ }^{3} P$ & 0.0429 & ${ }^{3} P$ & 0.0395 & ${ }^{3} P$ & 0.0365 & ${ }^{3} P$ & 0.0344 \\
\hline & diff. & -0.0002 & diff. & -0.0001 & diff. & 0.0006 & diff. & 0.0007 \\
\hline \multirow{3}{*}{ Bias } & ${ }^{1} P$ & 0.0186 & ${ }^{1} P$ & 0.0190 & ${ }^{1} P$ & 0.0181 & ${ }^{1} P$ & 0.0193 \\
\hline & ${ }^{3} P$ & 0.0271 & ${ }^{3} P$ & 0.0261 & ${ }^{3} P$ & 0.0259 & ${ }^{3} P$ & 0.0244 \\
\hline & diff. & -0.0085 & diff. & -0.0071 & diff. & -0.0078 & diff. & -0.0051 \\
\hline
\end{tabular}

The left hand sides of (3.1) and (3.2) are estimated by

$$
\begin{aligned}
E_{D_{n}}\left[\operatorname{Var}\left(\gamma \mid D_{n}\right)\right] & \approx \frac{1}{M} \sum_{j=1}^{M} \operatorname{Var}\left(\gamma \mid D_{n, j}\right), \\
E_{D_{n}}\left[l\left(D_{n}\right)\right] & \approx \frac{1}{M} \sum_{j=1}^{M} l\left(D_{n, j}\right) .
\end{aligned}
$$

In the numerical results presented here, we took $M=1000$. When $\theta=0.3$, Table 7 shows that with 6 patients, we can estimate the MTD with an average posterior standard deviation equal to $25 \%$ of the range of the dose and that a $17 \%$ decrease in the average posterior standard deviation is achieved when increasing the sample size from 6 to 40 patients. Similarly, the average length of the $90 \%$ HPD interval is $74 \%$ of the dose range when 6 patients are enrolled in the phase I trial and a reduction of $16 \%$ of this length is achieved when increasing the number of patients from 6 to 40. Figures 1 and 2 show the average posterior standard deviation and average lengths of the 95\% HPD intervals as functions of the sample size $n$ and target probability of DLT $\theta$.

\subsection{Illustrative Example}

A randomized phase I clinical trial of the combination bortezomib and melphalan as conditioning for autologous stem cell transplant in patients with multiple myeloma was designed using EWOC and the results published in [27]. patients are randomized to arm A where a fixed dose of melphalan $\left(100 \mathrm{mg} / \mathrm{m}^{2}\right)$ is given before bortezomib and arm B where the same fixed dose of melphalan is given after bortezomib. The doses available for bortezomib are $0.4,0.7,1.0,1.3$, and $1.6 \mathrm{mg} / \mathrm{m}^{2}$ with the first patient in either arm receiving $1.0 \mathrm{mg} / \mathrm{m}^{2}$. For each arm, the MTD is defined to be the dose level of bortezomib that when 
Table 7: Average posterior standard deviation and average length of HPD of the posterior distribution of the MTD that are achieved for a given sample size for $\theta=0.3$.

\begin{tabular}{lccc}
\hline$n$ & Mean SD & Length of $90 \%$ HPD & Length of $95 \%$ HPD \\
\hline 6 & 0.2453 & 0.7386 & 0.8161 \\
8 & 0.2399 & 0.7238 & 0.8040 \\
10 & 0.2351 & 0.7111 & 0.7925 \\
12 & 0.2309 & 0.6985 & 0.7818 \\
14 & 0.2281 & 0.6913 & 0.7755 \\
16 & 0.2248 & 0.6821 & 0.7678 \\
18 & 0.2221 & 0.6748 & 0.7608 \\
20 & 0.2197 & 0.6673 & 0.7546 \\
22 & 0.2176 & 0.6624 & 0.7500 \\
24 & 0.2153 & 0.6557 & 0.7439 \\
26 & 0.2136 & 0.6505 & 0.7395 \\
28 & 0.2119 & 0.6455 & 0.7352 \\
30 & 0.2102 & 0.6410 & 0.7313 \\
32 & 0.2085 & 0.6350 & 0.7257 \\
34 & 0.2072 & 0.6313 & 0.7221 \\
36 & 0.2057 & 0.6262 & 0.7176 \\
38 & 0.2050 & 0.6240 & 0.7162 \\
40 & 0.2036 & 0.6200 & 0.7123 \\
\hline
\end{tabular}

administered in combination with $100 \mathrm{mg} / \mathrm{m}^{2}$ of melphalan (either before or after) to a patient results in a probability equal to $\theta=0.33$ that a dose limiting toxicity will be manifest. In this trial, we start at $\alpha=0.3$ and increase $\alpha$ in small increments of 0.05 until $\alpha=0.5$, this value being a compromise between the therapeutic aspect of the Bortezomib and its toxic side effects. Since the doses in this trial are discrete, the dose allocated to the next patient is obtained by rounding down the dose recommended by EWOC algorithm to the nearest discrete dose, see $[9,15]$ on how to conduct a trial in the presence of a prespecified set of discrete doses.

Figure 3 shows all the possible dose sequences that could be realized for the first four patients, assuming that only one patient is treated at each dose and a selected situation for patient 5. The principal investigator (PI) wanted to determine the number of patients to accrue in each arm so that the posterior standard deviation of the MTD is no more than one-fifth the range of the dose level. This statistical constraint combined with the logistics such as availability of the resources for the PI, number of patients available, and limits on the duration of the trial leads us to select 20 patients per arm. In fact, a sample size of 20 results in an average posterior standard deviation $E_{D_{20}}\left[\left(\operatorname{Var}\left(\gamma \mid D_{20}\right)\right)^{1 / 2}\right] \approx 0.228$; This is just below one-fifth the range of dose levels 0.4-1.6.

\section{Concluding Remarks}

The objectives of this paper are to provide a rational for the choice of cohort sizes and number of patients to accrue in a phase I cancer clinical trial when the Bayesian adaptive design EWOC is used. In these trials, patients are typically enrolled in cohorts of size three for 


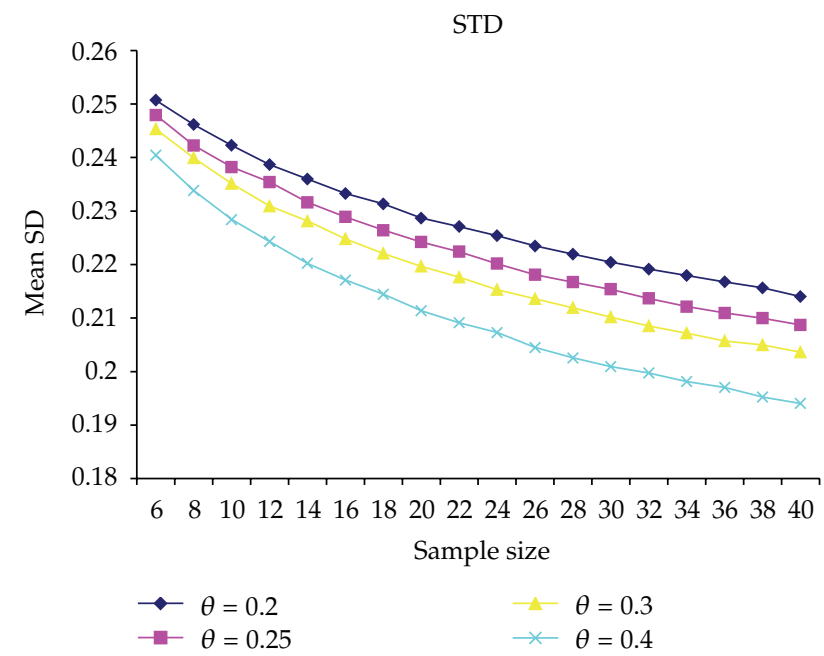

Figure 1: Estimated mean posterior standard deviation as a function of the number of patients accrued to the trial for different target probabilities of DLT $\theta$.

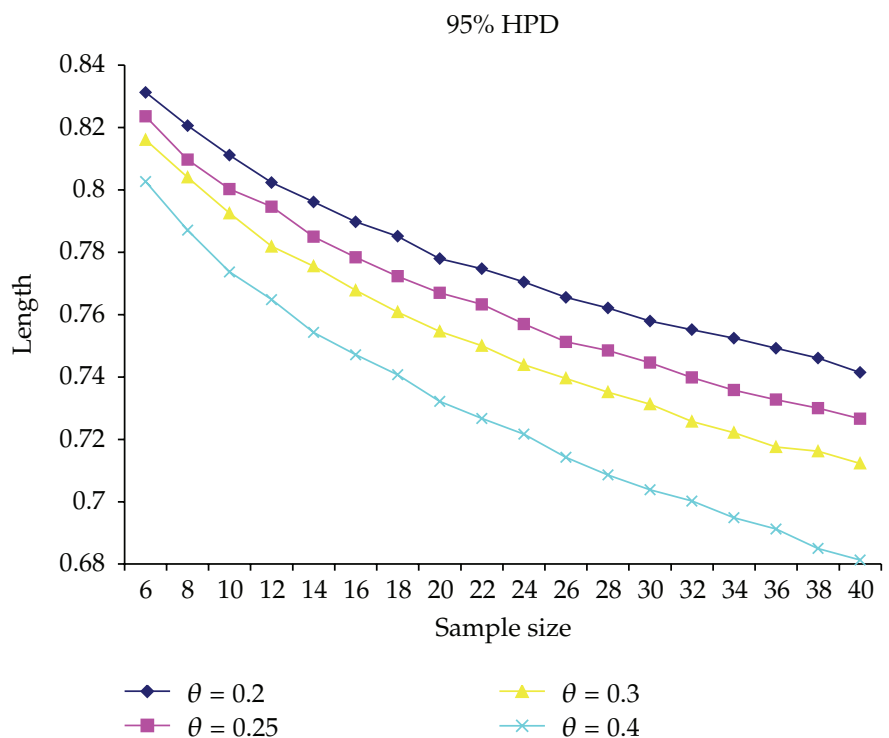

Figure 2: Estimated mean length of HPD of the posterior distribution of the MTD as a function of the number of patients accrued to the trial for different target probabilities of DLT $\theta$.

no apparent reason other than being in agreement with the traditional " $3+3$ " design and shortening the duration of the trial. We have shown through simulations that the two designs are equally safe and that no practical gain is achieved in terms of the efficiency of the estimate of the MTD. Depending on how important the length of the trial is to the clinician and the institution, we recommend using one patient per dose level to avoid seeing simultaneous toxic events when a group of patients is treated at the same dose level as was the case in 


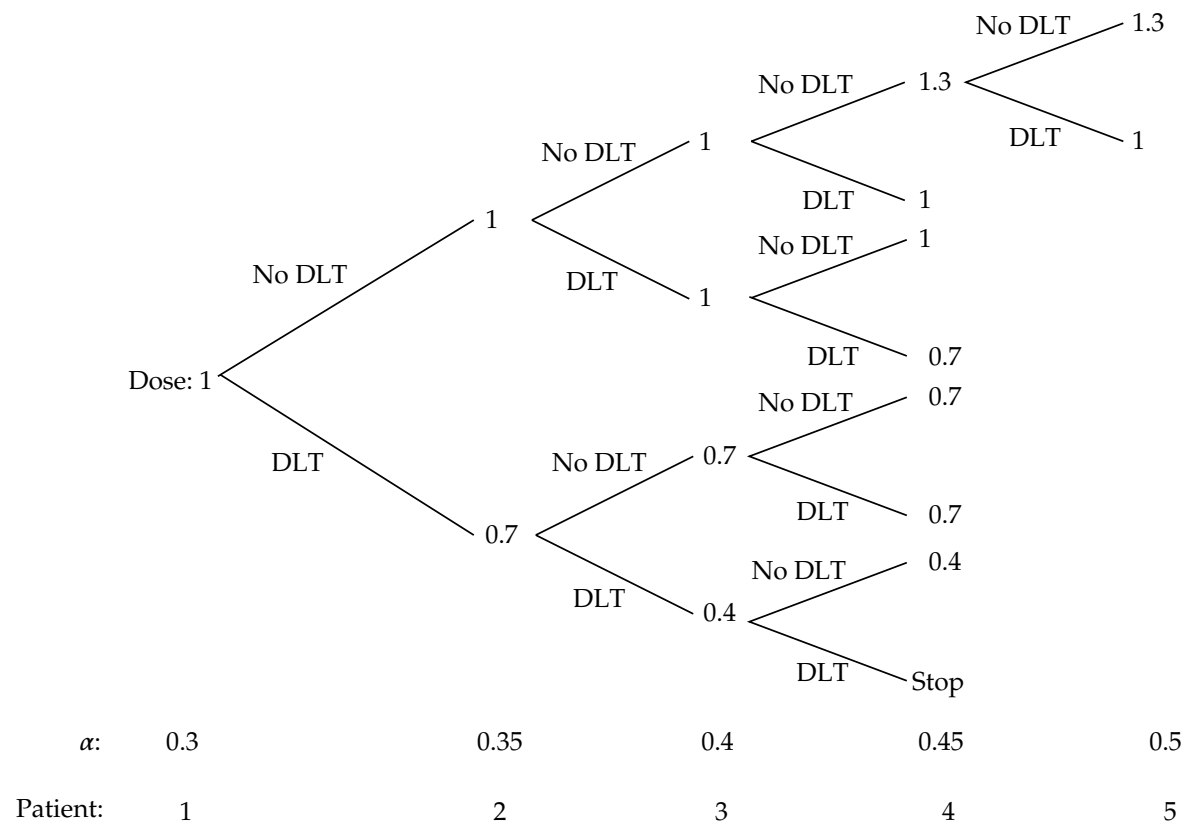

Figure 3: All the possible dose sequences that could be realized for the first four patients and a selected situation for patient 5 . It assumes no simultaneous treatment of patients.

a recent phase I trial of the drug TGN1412, see [32]. In that trial, six volunteers were given what was believed to be a safe dose of an anti-inflammatory drug TGN1412. Shortly after, all 6 were admitted into intensive care due to severe reactions including swelling of the head and neck.

The simulation results were obtained by generating the toxicity responses using the logistic model (2.3). This assumption may not be true in practice and the operating characteristics of EWOC may be sensitive to model misspecification. However, for the purpose of model comparisons between ${ }^{1} P$ and ${ }^{3} P$ designs, any model misspecification for the probability of toxicity response will affect the two designs the same way.

In the second part of the paper, we addressed the SSD problem by giving tabulated values of the number of patients to accrue in a cancer phase I clinical trial as a function of the posterior standard deviation and length of the HPD interval of the MTD on the average over all possible trials. Although this aspect of the trial never received much emphasis in the literature due to the relatively small number of patients and logistical issues associated with such trials, we felt that providing a measure of the accuracy of the estimate of the MTD that can be achieved for a given sample size would help the clinicians understand what can and cannot be achieved during this phase of the trial. Our results show that in general, there is $17 \%$ decrease in the average posterior standard deviation of the MTD when the sample size increases from 6 to 40 patients and that for a sample size of 20 patients, the average posterior standard deviation of the MTD is about one-fifth the range of the dose levels. Although this decrease in the average posterior standard deviation seems modest, we note that this is dependent upon the use of prior distribution for the MTD. A more informative prior based on past data will result in smaller average posterior standard deviations and narrower HPD intervals. 


\section{Acknowledgments}

This paper is supported in part by the National Center for Research Resources, Grant UL1RR033176, and is now at the National Center for Advancing Translational Sciences, Grant UL1TR000124 (M. Tighiouart and A. Rogatko), Grant 5P01CA098912-05 (A. Rogatko), and Grant P01 DK046763 (A. Rogatko). The content is solely the responsibility of the authors and does not necessarily represent the official views of the NIH.

\section{References}

[1] W. F. Rosenberger and L. M. Haines, "Competing designs for phase I clinical trials: a review," Statistics in Medicine, vol. 21, no. 18, pp. 2757-2770, 2002.

[2] N. Ting, "Dose finding in drug development," in Dose Finding in Drug Development, Springer, New York, NY, USA, 2006.

[3] J. O'Quigley, M. Pepe, and L. Fisher, "Continual reassessment method: a practical design for phase 1 clinical trials in cancer," Biometrics, vol. 46, no. 1, pp. 33-48, 1990.

[4] D. Faries, "Practical modifications of the continual reassessment method for phase I cancer clinical trials," Journal of Biopharmaceutical Statistics, vol. 4, no. 2, pp. 147-164, 1994.

[5] S. N. Goodman, M. L. Zahurak, and S. Piantadosi, "Some practical improvements in the continual reassessment method for phase I studies," Statistics in Medicine, vol. 14, no. 11, pp. 1149-1161, 1995.

[6] S. Moller, "An extension of the continual reassessment methods using a preliminary up-and-down design in a dose finding study in cancer patients, in order to investigate a greater range of doses," Statistics in Medicine, vol. 14, no. 9-10, pp. 911-922, 1995.

[7] S. Piantadosi, J. D. Fisher, and S. Grossman, "Practical implementation of a modified continual reassessment method for dose-finding trials," Cancer Chemotherapy and Pharmacology, vol. 41, no. 6, pp. 429-436, 1998.

[8] B. E. Storer, "An evaluation of phase I clinical trial designs in the continuous dose-response setting," Statistics in Medicine, vol. 20, no. 16, pp. 2399-2408, 2001.

[9] J. Babb, A. Rogatko, and S. Zacks, "Cancer Phase I clinical Trials: efficient dose escalation with overdose control," Statistics in Medicine, vol. 17, pp. 1103-1120, 1998.

[10] S. Zacks, A. Rogatko, and J. Babb, "Optimal Bayesian-feasible dose escalation for cancer phase I trials," Statistics and Probability Letters, vol. 38, no. 3, pp. 215-220, 1998.

[11] J. S. Babb and A. Rogatko, "Patient specific dosing in a cancer phase I clinical trial," Statistics in Medicine, vol. 20, no. 14, pp. 2079-2090, 2001.

[12] M. Tighiouart, A. Rogatko, and J. S. Babb, "Flexible Bayesian methods for cancer phase I clinical trials. Dose escalation with overdose control," Statistics in Medicine, vol. 24, no. 14, pp. 2183-2196, 2005.

[13] M. Tighiouart and A. Rogatko, "Dose finding in oncology—parametric methods," in Dose Finding in Oncology_Parametric Methods, N. Ting, Ed., Springer, New York, NY, USA, 2006.

[14] M. Tighiouart and A. Rogatko, "Dose escalation with overdose control," in Dose Escalation With Overdose Control, S. Chevret, Ed., John Wiley \& Sons, New York, NY, USA, 2006.

[15] M. Tighiouart and A. Rogatko, "Dose finding with escalation with overdose control (EWOC) in cancer clinical trials," Statistical Science, vol. 25, no. 2, pp. 217-226, 2010.

[16] E. Garrett-Mayer, "The continual reassessment method for dose-finding studies: a tutorial," Clinical Trials, vol. 3, no. 1, pp. 57-71, 2006.

[17] S. Zohar, A. Latouche, M. Taconnet, and S. Chevret, "Software to compute and conduct sequential Bayesian phase I or II dose-ranging clinical trials with stopping rules," Computer Methods and Programs in Biomedicine, vol. 72, no. 2, pp. 117-125, 2003.

[18] Z. Xu, M. Tighiouart, and A. Rogatko, "EWOC 2.0: interactive software for dose escalation in cancer phase I clinical trials," Drug Information Journal, vol. 41, no. 2, pp. 221-228, 2007.

[19] A. Rogatko, M. Tighiouart, and Z. Xu, "EWOC 3.1 application software," 2009, http:/ / biostatistics.csmc.edu/ewoc/index.php.

[20] S. Zohar and S. Chevret, "The continual reassessment method: comparison of Bayesian stopping rules for dose-ranging studies," Statistics in Medicine, vol. 20, no. 19, pp. 2827-2843, 2001.

[21] Y. Lin and W. J. Shih, "Statistical properties of the traditional algorithm-based designs for phase I cancer clinical trials," Biostatistics, vol. 2, pp. 203-215, 2001.

[22] A. Ivanova, "Escalation, group and A + B designs for dose-finding trials," Statistics in Medicine, vol. 25, no. 21, pp. 3668-3678, 2006. 
[23] H. Borghaei, K. Alpaugh, G. Hedlund et al., "Phase I dose escalation, pharmacokinetic and pharmacodynamic study of naptumomab estafenatox alone in patients with advanced cancer and with docetaxel in patients with advanced non-small-cell lung cancer," Journal of Clinical Oncology, vol. 27, no. 25, pp. 4116-4123, 2009.

[24] J. D. Cheng, J. S. Babb, C. Langer et al., “Individualized patient dosing in phase I clinical trials: the role of Escalation with Overdose Control in PNU-214936," Journal of Clinical Oncology, vol. 22, no. 4, pp. 602-609, 2004.

[25] G. M. Freedman, N. J. Meropol, E. R. Sigurdson et al., "Phase I trial of preoperative hypofractionated intensity-modulated radiotherapy with incorporated boost and oral capecitabine in locally advanced rectal cancer," International Journal of Radiation Oncology Biology Physics, vol. 67, no. 5, pp. 1389-1393, 2007.

[26] N. Haas, B. Roth, C. Garay et al., "Phase I trial of weekly paclitaxel plus oral estramustine phosphate in patients with hormone-refractory prostate cancer," Urology, vol. 58, no. 1, pp. 59-64, 2001.

[27] S. Lonial, J. Kaufman, M. Tighiouart et al., "A phase I/II trial combining high-dose melphalan and autologous transplant with bortezomib for multiple myeloma: a dose- and schedule-finding study," Clinical Cancer Research, vol. 16, no. 20, pp. 5079-5086, 2010.

[28] R. J. Schilder, J. M. Gallo, M. M. Millenson et al., "Phase I trial of multiple cycles of high-dose carboplatin, paclitaxel, and topotecan with peripheral-blood stem-cell support as front-line therapy," Journal of Clinical Oncology, vol. 19, no. 4, pp. 1183-1194, 2001.

[29] R. Sinha, J. L. Kaufman, N. King et al., "A phase 1 dose escalation study of bortezomib combined with rituximab, cyclophosphamide, doxorubicin, modified vincristine, and prednisone for untreated follicular lymphoma and other low-grade B-cell lymphomas," Cancer, vol. 118, no. 14, pp. 3538-3548, 2012.

[30] C. J. Adcock, "Sample size determination: a review," Journal of the Royal Statistical Society D, vol. 46, no. 2, pp. 261-283, 1997.

[31] L. Joseph, D. B. Wolfson, and R. Duberger, "Sample-size calculations for binomial proportions via highest posterior density intervals," Statistician, vol. 44, pp. 143-154, 1995.

[32] S. Senn, D. Amin, R. A. Bailey et al., "Statistical issues in first-in-man studies," Journal of the Royal Statistical Society A, vol. 170, pp. 517-579, 2007. 


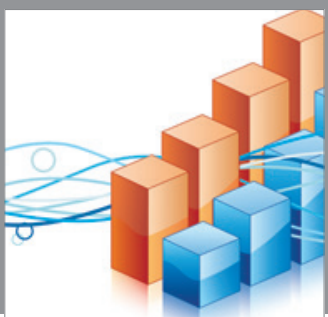

Advances in

Operations Research

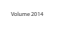

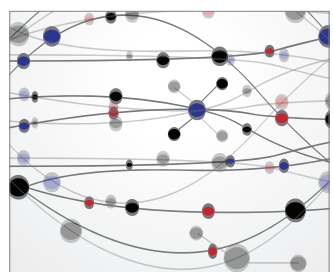

\section{The Scientific} World Journal
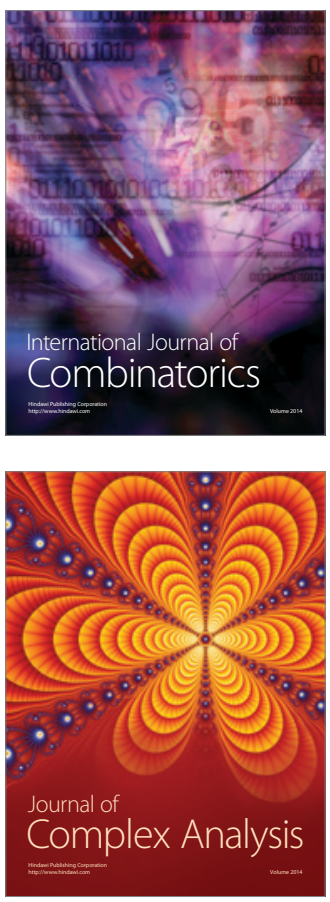

International Journal of

Mathematics and

Mathematical

Sciences
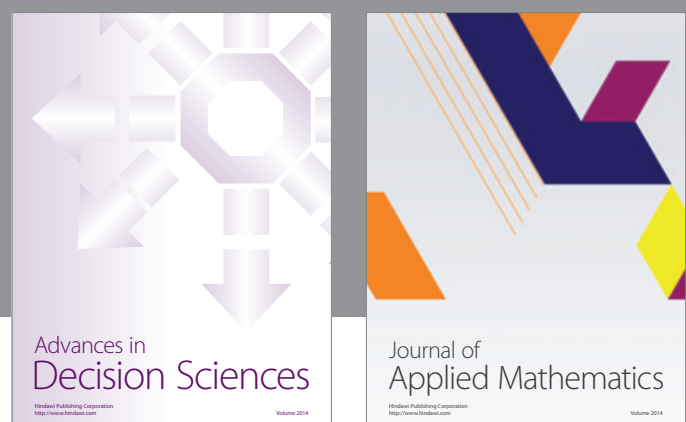

Journal of

Applied Mathematics
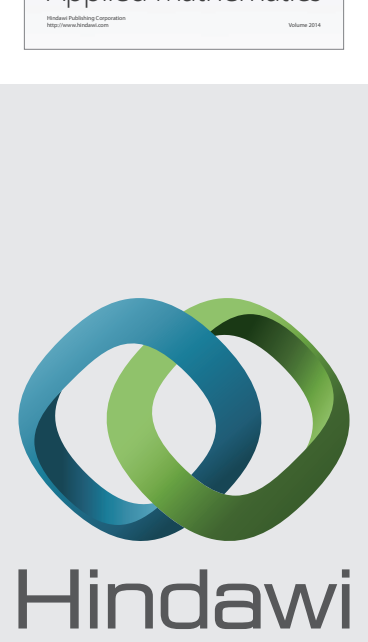

Submit your manuscripts at http://www.hindawi.com
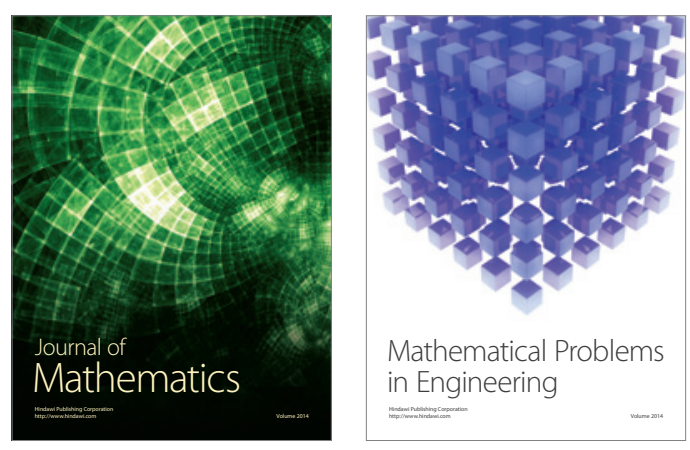

Mathematical Problems in Engineering
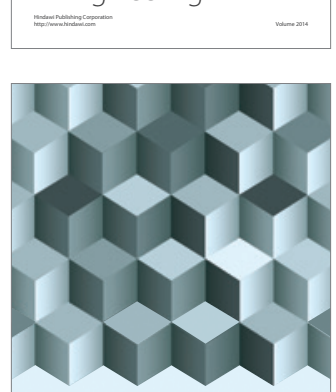

Journal of

Function Spaces
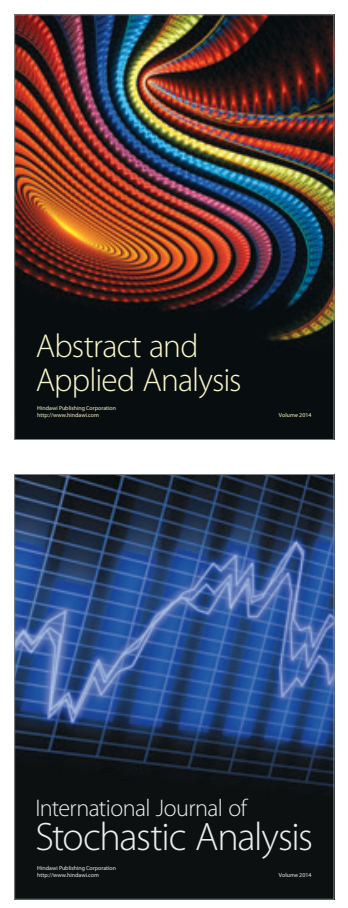

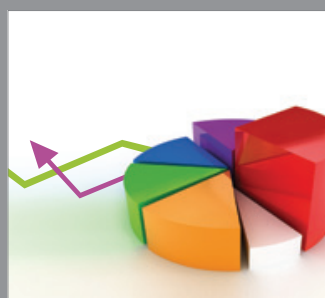

ournal of

Probability and Statistics

Promensencen
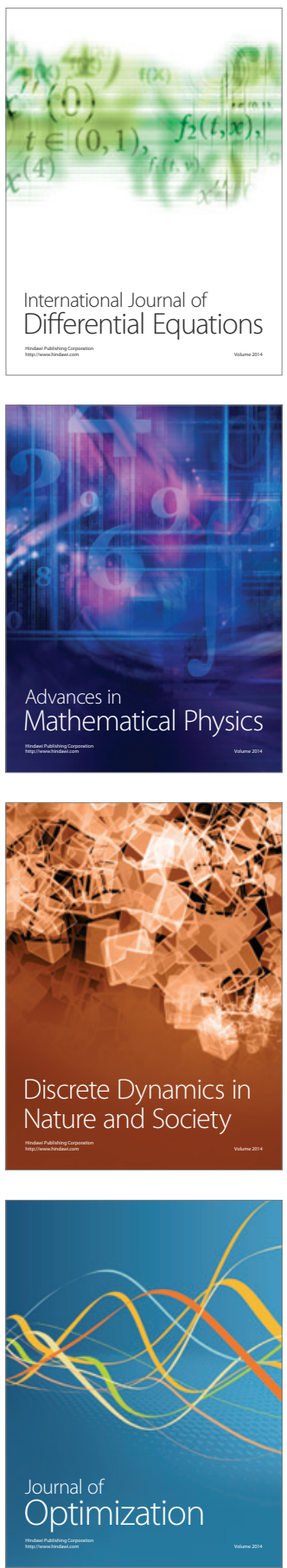